\title{
Hierarchical-environment-assisted non-Markovian and its effect on thermodynamic properties
}

Yongwei $\mathrm{Li}^{1}$ and $\mathrm{Lei}_{\mathrm{Li}}{ }^{* *}$ (1)

"Correspondence: lilei@imu.edu.cn

'School of Physical Science and

Technology, Inner Mongolia

University, Hohhot 010021, China

\begin{abstract}
We consider a microscopic collision model, i.e., a quantum system interacts with a hierarchical environment consisting of an auxiliary system and a reservoir. We show how the non-Markovian character of the system is influenced by the coupling strength of system-auxiliary system and auxiliary system-reservoir, coherence of environment and initial system-environment correlations. And we study the non-Markovianity induced by coherence of environment from the perspective of energy, further the relationship between information backflow and energy flux is obtained. Then we study the effect of non-Markovianity on thermodynamic properties. By studying the entropy change of system especially that from heat exchanges with the environment, we reveal the essence of entropy change between positive and negative values during non-Markovian evolution is due to the contribution of heat flux induced by coherence. And compared with the case of Markovian dynamics, we observe that the entropy production decreases in some specific time intervals under non-Markovian dynamics induced by the coupling strength. And this is different to the case of non-Markovianity caused by initial system-environment correlation, that we show the possibility of positive entropy production during the whole dynamics.
\end{abstract}

Keywords: Open quantum systems; Collision-based models; Quantum non-Markovianity; Quantum coherence; Initial correlation; Quantum thermodynamics

\section{Introduction}

The study of open quantum systems is of great importance in quantum information and computation recently. Because the dynamics of open quantum systems is greatly affected by its environment, and the environments are often very complex, solving the dynamics of open quantum systems has always been a challenge. The Markovian approximation is important to describe the dynamics of open quantum system either in terms of maps and Kraus operators or in terms of master equations [1]. One advantage of this approximation is that the dynamics of the system will be a Markovian process and can be described by a standard Markovian master equation.

(c) The Author(s) 2021. This article is licensed under a Creative Commons Attribution 4.0 International License, which permits use, sharing, adaptation, distribution and reproduction in any medium or format, as long as you give appropriate credit to the original author(s) and the source, provide a link to the Creative Commons licence, and indicate if changes were made. The images or other third party material in this article are included in the article's Creative Commons licence, unless indicated otherwise in a credit line to the material. If material is not included in the article's Creative Commons licence and your intended use is not permitted by statutory regulation or exceeds the permitted use, you will need to obtain permission directly from the copyright holder. To view a copy of this licence, visit http://creativecommons.org/licenses/by/4.0/. 
However, it has been shown that the Markovian approximation fails in many situations [2-5], and the non-Markovian dynamics have been received considerable attention and have been extensively studied recently [6-13]. Based on this, several measures of nonMarkovianity(NM) have been proposed [14-16]. With the help of these measures, one can claim that an evolution is non-Markovian if a nonzero degree of NM is detected. These measures have been applied to many models to investigate their non-Markovian characteristics [17-24]. Furthermore, the transition from Markovian to non-Markovian dynamics has also been theoretically and experimentally implemented based on these measures [25-31]. For example, Brito et al. have implemented the transitions from Markovianity to NM by preparing different system initial states or dynamically manipulating the subsystem coupling [26]. Ma et al. have showed how the non-Markovian character of the system is influenced by the coupling strength between the qubit and cavity and the correlation time of the reservoir, and they have found a phenomenon whereby the qubit Markovian and nonMarkovian transition exhibits a anomalous pattern in a parameter space depicted by the coupling strength and the correlation time of the reservoir [27]. In Ref. [31], initial systemenvironment correlations have been showed to substantially increase the distance between two qubit states evolving to long-time-limit states according to exact non-Markovian dynamics. And in Ref. [32], it have showed that the trace distance between two states of the open system can increase above its initial value when the system and its environment are initially correlated. In particular, Smirne et al. [33] have provided experimental evidence of the behavior showed in Ref. [32]. All of these factors together make it difficult to understand their independent role in the non-Markovian dynamics of open quantum system. However we have not seen any reports about the effect of coherence of environment on the non-Markovian dynamics. Thus an interesting question concerns how the independent role of these factors to influence the system dynamics, specifically systemenvironment coupling, initial system-environment correlations and the intraenvironment coherence.

As one of the representative models for studying open quantum systems, collision model, also called repeated interaction framework, has been extensively studied during recent decades [34-40]. A quantum collision model is a microscopic framework to describe the open dynamics of a system interacting with a reservoir assumed to consist of a large collection of smaller constituents (ancillas), and the system is assumed to interact (collide) sequentially with an ancilla at each time step [34, 41, 42]. It offers a bottom-top description of an environment, where one has precise theoretical control of the microscopic aspects that give rise to macroscopic characteristics of the reservoir. The collision model has been applied in non-Markovian dynamics widely [43-57]. For example, Ciccarello et al. have endowed the reservoir with memory by introducing interancillary collisions between next system-ancilla interactions [44]. Bernardes et al. have investigated the Markovian to nonMarkovian transitions in collision models by introducing correlations in the state of the environment [46]. In Ref. [47], the use of collision model with interenvironment swaps has displayed a signature of strongly non-Markovian dynamics that is highly dependent on the establishment of system-environment correlations. Campbell et al. have also identified the relevant system-environment correlations that lead to a non-Markovian evolution in a collision model [52]. Kretschmer et al. have studied the applicability of collisional models for non-Markovian dynamics of open quantum systems, and they have discussed the possibility to embed non-Markovian collision model dynamics into Markovian collision model 
dynamics in an extended state space [48]. Lorenzo et al. have shown that the composite quantum collision models they studied can accommodate some known relevant instances of non-Markovian dynamics [53]. In Ref. [54], a non-Markovian dynamics is established under a structured environment based on collision model. In Ref. [56], it has studied the effects of different strategies of system-environment interactions and states of the blocks on the non-Markovianities by introducing a block (a number of environment particles) as the unit of the environment instead of a single particle. Ref. [57] has found that the information is scrambled if the memory and environmental particles are alternatively squeezed along two directions which are perpendicular to each other.

Recently, the relation between NM and thermodynamics in open quantum system has attracted considerable attention. In Ref. [58], the heat flux has exhibited a nonexponential time behavior in the case of non-Markovian dynamics of the subsystem. In Ref. [59], the heat flux changes between positive to negative values for a non-Markovian evolution of the subsystem, which leads to a violation of open-system formulation of Landauer's principle for the heat and entropy fluxes. A similar result has also been obtained in Refs. $[60,61]$ that the Landauer's principle is violated in non-Markovian dynamics. Raja et al. have investigated how memory effects influence the ability to perform work on the driven qubit, and they have showed that the average work performed on the qubit can be used as a diagnostic tool to detect the presence or absence of memory effects [29]. Abiuso et al. have found that the non-Markovian effects can fasten the control and improve the power output of a quantum thermal engine [62]. Pezzutto et al. have addressed the effects that $\mathrm{NM}$ of the open-system dynamics of the work medium can have on the efficiency of the thermal machine [63]. Katz et al. have studied the performance characteristics of a heat rectifier and a heat pump in a non-Markovian framework [64]. Ref. [65] has studied the effects of environmental temperature on the NM of an open quantum system by virtue of collision models.

As one of the important thermodynamic quantities, the entropy production and the associated entropy production rates are crucial in the thermodynamic characterization of a given process. And the exploration of the relation between NM and entropy production has provoked great interest recently [66-73]. Refs. $[68,69]$ have shown that the entropy production can become transiently negative in the non-Markovian dynamics compared with the Markovian case, and the transient negativity of the entropy production rate is a sufficient sign of NM [69]. Further, Strasberg et al. have explored the link between a negative entropy production rate and NM precisely by showing under which conditions a negative entropy production rate implies NM and when it does not [70]. And Ref. [71] has shown that the possibility of positive entropy production rate with the initial correlation between the system and its heat reservoir.

In this paper, we consider a two-level system coupled to a structured environment consisting of a auxiliary system and a reservoir, and the reservoir is of a large collection of initially uncorrelated systems which we call ancillas (see Fig. 1). Based on this structured environment model, there can be different factors to influence the non-Markovian character of the system, and we mainly consider the effects of coherence of environment and initial system-environment correlations on system dynamics. And we study the relationship between NM and thermodynamic properties. For example, information backflow and energy flux, non-Markovian dynamics and entropy change of system, including entropy 
flux (entropy change of system induced by heat exchanges with environment) and entropy production.

\section{Methods}

\subsection{Model and solution}

We consider a qubit (system $S$ ) couples to a hierarchical environment, which contains a auxiliary qubit $A_{Q}$ and a collection of $N$ identical noninteracting ancillas (qubits) $\left\{\mathcal{R}_{1}, \mathcal{R}_{2}, \ldots, \mathcal{R}_{N}\right\}$ that consists a reservoir $\mathcal{R}$, and this reservoir is in the product state $\eta_{\text {tot }}=\otimes_{j=1}^{N} \eta_{j}$. In this way, the auxiliary qubit $A_{Q}$ and the reservoir hierarchically constitute the whole big reservoir $E$, which is called the environment of system $S$. And the general scheme is illustrated in Fig. 1. The Hamiltonians of system and a generic environment particle $E_{j}$ including the auxiliary qubit and ancillas are

$$
\hat{H}_{S(E)}=\omega_{S(E)} \hat{\sigma}_{z} / 2
$$

where $\hat{\sigma}_{z}$ is the Pauli matrices and we set $\hbar=1$ throughout this paper.

The evolution of system $S$ and its interaction with the environment are proceeded as follows. $S$ interacts with the environment first: Specifically $S$ and $A_{Q}$ interact and then $A_{Q}$ collides with the individual ancilla of the reservoir. As the assumption of a big reservoir $\mathcal{R}$ that $A_{Q}$ never interacts twice with the same ancilla, i.e., at each collision the state of the ancilla is refreshed. And this process is implemented through the unitary operator

$$
\hat{\mathcal{U}}_{\mathrm{SE}}=\hat{V}_{A_{Q}, \mathcal{R}_{j}} \hat{U}_{S, A_{Q}},
$$

where $\hat{U}_{S, A_{Q}}=e^{-i \hat{H}_{S, A}^{\text {int }} Q^{\tau}}, \hat{V}_{A_{Q}, \mathcal{R}_{j}}=e^{-i \hat{H}_{A_{Q}}^{\text {int }}, \mathcal{R}_{j}} \tau$. Here $\hat{H}_{S, A_{Q}}^{\text {int }}$ and $\hat{H}_{A_{Q}, \mathcal{R}_{j}}^{\text {int }}$ are the interaction between ' $S-A_{Q}$ ', ' $A_{Q}-\mathcal{R}_{j}$ ' respectively, and $\tau$ is the interaction time.

In our model, we consider a coherent interaction between the bipartite systems including ' $S-A_{Q}$ ' and ' $A_{Q}-\mathcal{R}_{j}$, , i.e., a mechanism that can be described by a Hamiltonian model of some form, specifically in this paper we suppose that the interaction Hamiltonian is

$$
\begin{aligned}
\hat{H}_{S, A_{Q}\left(A_{Q}, \mathcal{R}_{j}\right)}^{\mathrm{int}}= & g_{1(2)}\left(\hat{\sigma}_{x}^{S\left(A_{Q}\right)} \hat{\sigma}_{x}^{A_{Q}\left(\mathcal{R}_{j}\right)}+\hat{\sigma}_{y}^{S\left(A_{Q}\right)} \hat{\sigma}_{y}^{A_{Q}\left(\mathcal{R}_{j}\right)}\right. \\
& \left.+\hat{\sigma}_{z}^{S\left(A_{Q}\right)} \hat{\sigma}_{z}^{A_{Q}\left(\mathcal{R}_{j}\right)}\right)
\end{aligned}
$$

Figure 1 Sketch of the protocol of system $S$ plus a hierarchical environment. $S$ interacts with the environment: After $A_{Q}$ interacts with $\mathcal{R}_{n}$ (the $n$th ancilla of reservoir $\mathcal{R}$ ), it collides with $S$ and is then directed to $\mathcal{R}_{n+1}$

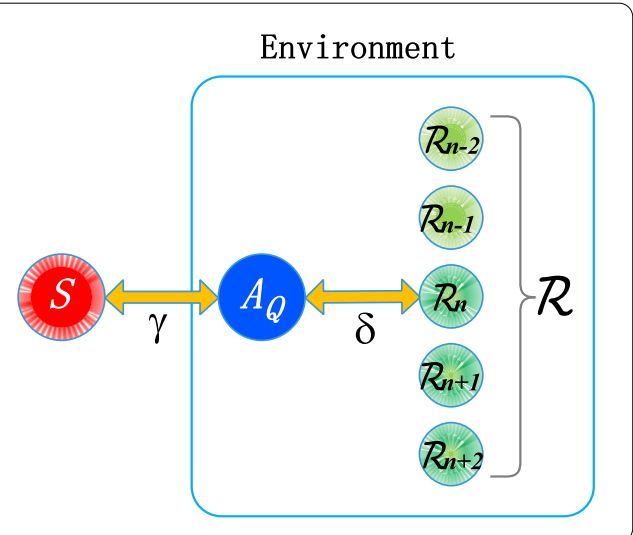


where $\hat{\sigma}_{i}^{S}, \hat{\sigma}_{i}^{A_{Q}}$ and $\hat{\sigma}_{i}^{\mathcal{R}_{j}}(i=x, y, z)$ are the Pauli matrices, and $g_{1(2)}$ is a coupling constant. And we use the result [34]

$$
e^{i \frac{\phi}{2}\left(\hat{\sigma}_{x} \otimes \hat{\sigma}_{x}+\hat{\sigma}_{y} \otimes \hat{\sigma}_{y}+\hat{\sigma}_{z} \otimes \hat{\sigma}_{z}\right)}=e^{-i \frac{\phi}{2}}\left(\cos \phi \hat{\mathbb{I}}+i \sin \phi \hat{S}^{\mathrm{sw}}\right),
$$

where $\hat{\mathbb{I}}$ is the identity operator, and $\hat{S}^{\text {sw }}$ is the two-particle swap operator, i.e., it is the unitary operation whose action is $\left|\psi_{1}\right\rangle \otimes\left|\psi_{2}\right\rangle \rightarrow\left|\psi_{2}\right\rangle \otimes\left|\psi_{1}\right\rangle$ for all $\left|\psi_{1}\right\rangle,\left|\psi_{2}\right\rangle \in \mathbb{C}^{2}$. We can now write the unitary time-evolution operator $\hat{U}_{S, A_{Q}}$ in Eq. (2) as

$$
\hat{U}_{S, A_{Q}}(\gamma)=(\cos \gamma) \hat{\mathbb{I}}_{S, A_{Q}}+i(\sin \gamma) \hat{S}_{S, A_{Q}}^{\mathrm{sw}}
$$

where $\gamma=2 g_{1} \tau$ is a dimensionless interaction strength. And when $\gamma=0$ Eq. (5) is reduced into an identity operator and indicates that there is no interaction between $S$ and $A_{Q}$; and when $\gamma=\pi / 2$ Eq. (5) is reduced into a fully swap operator and represents a complete exchange of quantum state information between $S$ and $A_{Q}$. Thus in the range of $\gamma \in[0, \pi / 2]$, the larger the $\gamma$, the stronger the coupling. And in the ordered basis $\{|00\rangle,|01\rangle,|10\rangle,|11\rangle\}$, $\hat{S}_{S, A_{Q}}^{\mathrm{sw}}$ in Eq. (5) reads [74]

$$
\hat{S}_{S, A_{Q}}^{\mathrm{SW}}=\left(\begin{array}{cccc}
1 & 0 & 0 & 0 \\
0 & 0 & 1 & 0 \\
0 & 1 & 0 & 0 \\
0 & 0 & 0 & 1
\end{array}\right) .
$$

Similarly $\hat{V}_{A_{Q}, \mathcal{R}_{j}}$ in Eq. (2) can be written as

$$
\hat{V}_{A_{Q}, \mathcal{R}_{j}}(\delta)=(\cos \delta) \hat{\mathbb{I}}_{A_{Q}, \mathcal{R}_{j}}+i(\sin \delta) \hat{S}_{A_{Q}, \mathcal{R}_{j}}^{\mathrm{sw}},
$$

with $\delta \neq \gamma$, in general, and the analog of the operations introduced above applies to $\hat{\mathbb{I}}_{A_{Q}, \mathcal{R}_{j}}$ and $\hat{S}_{A_{Q}, \mathcal{R}_{j}}^{\text {sw }}$ (swap gate between $A_{Q}$ and $\mathcal{R}_{j}$ ). As mentioned above the dynamics of system $S$ consists of sequential system-environment interaction and each step is treated in the following process: First $S$ and $A_{Q}$ interact and then subsequently $A_{Q}$ collides with $\mathcal{R}_{j}$ (one of the ancillas in $\mathcal{R}$ ). Thus the system is brought from step $n$ to step $n+1$ through the process

$$
\rho_{n}^{S, A_{Q}} \otimes \eta_{n+1} \rightarrow \rho_{n+1}^{\mathrm{SE}}=\hat{\mathcal{U}}_{\mathrm{SE}}\left(\rho_{n}^{S, A_{Q}} \otimes \eta_{n+1}\right) \hat{\mathcal{U}}_{\mathrm{SE}}^{\dagger}
$$

where $\rho_{n}^{S, A_{Q}}$ is the state of ' $S-A_{Q}$ ' after the $n$th interaction. Hence after the $(n+1)$ th interaction, we can obtain the reduced system state, $\rho_{n+1}^{S, A_{Q}}=\operatorname{Tr}_{\mathcal{R}}\left[\rho_{n+1}^{\mathrm{SE}}\right]$ (the state of ' $S-A_{Q}$ '), $\rho_{n+1}^{S}=\operatorname{Tr}_{A_{Q}}\left[\rho_{n+1}^{S, A_{Q}}\right]$ (the state of $S$ ) and $\rho_{n+1}^{A_{Q}}=\operatorname{Tr}_{S}\left[\rho_{n+1}^{S, A_{Q}}\right]$ (the state of $A_{Q}$ ), where $\operatorname{Tr}_{x}[\cdots]$ means the trace of $x$ degree of freedom.

\subsection{NM}

The trace distance between two quantum states is one of the most important measures of distinguishability of quantum states [74], which is given by

$$
\mathcal{D}\left(\rho_{1}, \rho_{2}\right)=\frac{1}{2} \operatorname{Tr}\left|\rho_{1}-\rho_{2}\right|,
$$


where $|A|=\sqrt{A^{\dagger} A}$ for any operator $A$. It is obvious that for any pair of states $\rho_{1}$ and $\rho_{2}$ the trace distance satisfies the inequality $0 \leq \mathcal{D}\left(\rho_{1}, \rho_{2}\right) \leq 1$. For the time evolution of a quantum state described by a trace-preserving completely positive map, the trace distance is always less than or equal to the initial value [75]; that is,

$$
\mathcal{D}\left(\rho_{1}(t), \rho_{2}(t)\right) \leq \mathcal{D}\left(\rho_{1}(0), \rho_{2}(0)\right) .
$$

In particular, for a Markovian evolution it can always be represented by a dynamical semigroup of completely positive and trace-preserving maps [76], and we obtain the inequality

$$
\mathcal{D}\left(\rho_{1}(t+\tau), \rho_{2}(t+\tau)\right) \leq \mathcal{D}\left(\rho_{1}(t), \rho_{2}(t)\right)
$$

for any positive $\tau$, which indicates that the trace distance decreases monotonically with time. The decrease of trace distance corresponds to the reduction of distinguish ability between the two states, and this could be interpreted as an outflow of information from the system to the environment. In contrast to this, if the time derivative of the trace distance becomes positive in some time intervals, the time evolution is non-Markovian [14, 17]. Furthermore, if the trace distance exceeds the initial value, the time evolution cannot be described by a trace-preserving completely positive map. Based on this, a measure of NM can be defined by [14]

$$
\mathcal{N}=\max _{\rho_{1}(0), \rho_{2}(0)} \int_{\sigma>0} \mathrm{~d} t \sigma\left(t, \rho_{1}(0), \rho_{2}(0)\right)
$$

where $\sigma\left(t, \rho_{1}(0), \rho_{2}(0)\right)=\frac{\mathrm{d}}{\mathrm{d} t} \mathcal{D}\left(\rho_{1}(t), \rho_{2}(t)\right)$. Conceptually, $\mathcal{N}$ accounts for all regions where the distance between two arbitrary input states increases, thus witnessing a backflow of information from the environment to system. And in this case, an evolution is nonMarkovian if and only if $\mathcal{N}>0$.

As the evolution in our model proceeds in discrete steps, we will employ the discretized version of Eq. (12), which is obtained as [17, 77]

$$
\mathcal{N}=\max \sum_{n \in \sigma^{+}}\left[\mathcal{D}\left(\rho_{1, n+1}, \rho_{2, n+1}\right)-\mathcal{D}\left(\rho_{1, n}, \rho_{2, n}\right)\right]
$$

with $\sigma^{+}=\bigcup_{n}(n, n+1)$ is the union of all the interaction steps $(n, n+1)$ within which $\mathcal{D}\left(\rho_{1, n+1}, \rho_{2, n+1}\right)-\mathcal{D}\left(\rho_{1, n}, \rho_{2, n}\right)>0$, and $\left\{\rho_{1, n+1}, \rho_{2, n+1}\right\}$ a pair of state of system obtained starting from the corresponding pair of orthogonal state $\left\{\left|\psi_{+}\right\rangle,\left|\psi_{-}\right\rangle\right\}$after $n+1$ steps of our protocol,

$$
\begin{aligned}
& \left|\psi_{+}\right\rangle=\cos \frac{\theta}{2}|0\rangle+e^{i \varphi} \sin \frac{\theta}{2}|1\rangle, \\
& \left|\psi_{-}\right\rangle=\sin \frac{\theta}{2}|0\rangle-e^{i \varphi} \cos \frac{\theta}{2}|1\rangle,
\end{aligned}
$$

where $\theta \in\left[0, \frac{\pi}{2}\right]$ and $\varphi \in[0,2 \pi]$. The maximization in Eq. (13) performed over all possible values of $\theta$ and $\varphi$, i.e., all possible orthogonal pairs of initial system states. 


\section{Results and discussion}

\subsection{Non-Markovian dynamics of the system}

In this section we study how the system dynamics can be affected by different ways, including the coupling strength between the bipartite systems (' $S-A_{Q}$ ' and ' $A_{Q}-\mathcal{R}$ '), coherence of the environment and initial system-environment correlation. We consider the initial state of each ancilla of reservoir $\mathcal{R}$ as

$$
\rho_{E}=p|\psi\rangle\langle\psi|+(1-p) \rho_{\beta}
$$

where $p \in[0,1],|\psi\rangle=\frac{1}{\sqrt{Z}}\left(e^{-\frac{1}{4} \omega_{E} \beta}|0\rangle+e^{i \phi_{1}+\frac{1}{4} \omega_{E} \beta}|1\rangle\right)$ with a relative phase $\phi_{1}$, and $\rho_{\beta}$ is the thermal state assumed to be of canonical equilibrium form, i.e., $\rho_{\beta}=\frac{1}{Z} e^{-\beta \hat{H}_{E}}$. Here $\beta=$ $1 / T$ and $Z=\operatorname{Tr}\left[e^{-\beta \hat{H}_{E}}\right]$ are the inverse temperature and the partition function respectively. Note that the diagonal elements of states $\rho_{E}$ and $\rho_{\beta}$ are identical, and compared with the thermal state, the off-diagonal elements of state $\rho_{E}$ are nonzero if $p \neq 0$. Therefore, Eq. (15) can also be written as

$$
\rho_{E}=\rho_{\beta}+p \rho_{\text {coh }},
$$

where $\rho_{\text {coh }}$ is the non-diagonal part of state $|\psi\rangle\langle\psi|$, i.e., the off-diagonal elements of $\rho_{\text {coh }}$ are the same as that of state $|\psi\rangle\langle\psi|$ and the diagonal elements are zero.

\subsubsection{Effect of the coupling strength on NM}

We suppose that the environment is in thermal state, i.e., all environment particles including $A_{Q}$ and each ancilla are in the state $\rho_{\beta}$ with $T=\omega_{E}=1$. We numerically calculate the degree of NM for different $\gamma$ and $\delta$ which is presented in Fig. 2. We can see that the whole diagram is divided into two regions, where the green stars represent the degree of NM being equal to zero (Markovian region) and the red dots represent the degree of NM being larger than zero (non-Markovian region). It shows that the non-Markovian dynamics of the system is determined by a delicate balance between the two parameters $\gamma$ and $\delta$. Specifically the system dynamics is Markovian for small $\gamma$ and larger $\delta$, and the nonMarkovian region increases with the increase of $\gamma$. Physically this can be understood as following. When the interaction between $S$ and $A_{Q}$ is small (small $\gamma$ ) and with a relatively large interaction between $A_{Q}$ and $\mathcal{R}_{j}$ (larger $\delta$ ), the information obtained by $A_{Q}$ from $S$ is

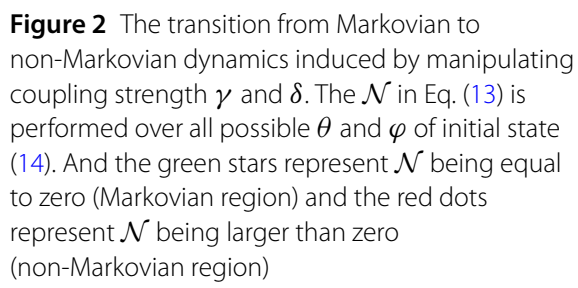


less and all of which flow into the reservoir $\mathcal{R}$, which forms Markovian dynamics of the system. In other words, the system is losing information at a slower rate than that of the evolution of environment, thus the backflow of information cannot happen now. However with the increase of $\gamma$, more and more information flows from $S$ into $A_{Q}$ which leads to only part of the information flows into the reservoir and the rest is reserved and flows back to $S$, and in this case the non-Markovian dynamics of system is formed.

\subsubsection{Effects of coherence of environment on NM and energy flux}

We consider the case of environment with coherence, i.e., $A_{Q}$ and each ancilla are in state (16) with a relative phase $\phi_{2}$ and $\phi_{1}$ respectively. Thus the phase difference between reservoir $\mathcal{R}$ and $A_{Q}$ is $\phi=\phi_{1}-\phi_{2}$. In Fig. 3, we plot the variation of the NM with respect to $p$ for fixed $\phi(\phi=0)$ (Fig. 3(a)), and $\phi$ for fixed $p(p=0.4)$ (Fig. 3(b)), and the coupling strength $\gamma$ and $\delta$ are of the Markovian region of coupling presented in Fig. 2. From numerical calculations we find that the system dynamics is Markovian for $p \in[0,0.4]$, and in the region $p \in(0.4,1]$ the increase of $p$ leads to an increase of NM. An interesting feature here is that a transition from Markovian to non-Markovian dynamics is observed. Besides parameter $p$ phase difference $\phi$ is also one of the influence factor of coherence of environment. The system dynamics is Markovian for $\phi \in[0, \pi / 4]$, and in the region $\phi \in(\pi / 4, \pi]$ the increase of $\phi$ leads to an increase of NM, in the region $\phi \in[0,2 \pi]$ the change of NM is symmetrical about $\phi=\pi$. Also a transition from Markovian to non-Markovian dynamics is observed by means of $\phi$. Physically this can be understood as following. As we consider energyconserving interactions, i.e., $\left[\hat{H}_{S, A_{Q}}^{\text {int }},\left(\hat{H}_{S}+\hat{H}_{A_{Q}}\right)\right]=0,\left[\hat{H}_{A_{Q}, \mathcal{R}_{j}}^{\text {int }},\left(\hat{H}_{A_{Q}}+\hat{H}_{\mathcal{R}_{j}}\right)\right]=0$, leading to

$$
\Delta E_{n}=-Q_{A_{Q}, \mathcal{R}_{n}},
$$

where $\Delta E_{n}=\operatorname{Tr}\left\{\hat{H}_{S}\left[\rho_{S}(n+1)-\rho_{S}(n)\right]\right\}$, is the change in energy of the system in each interaction, and $Q_{A_{Q}, \mathcal{R}_{n}}$ is the change in energy of the environment. From Eq. (16), compared to initial thermal state of each element of the environment, coherence, i.e., the second term in Eq. (16), is added, and the amount of coherence increases with the increase of
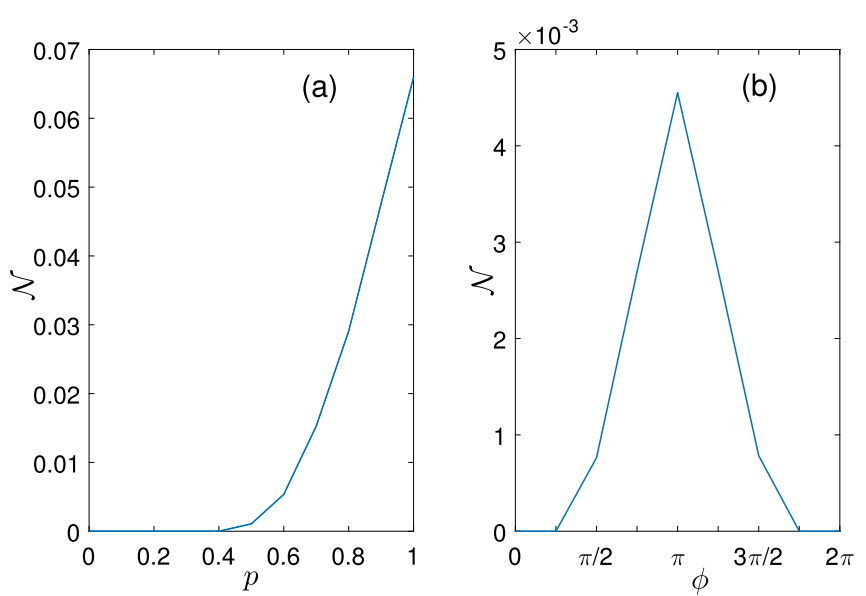

Figure 3 Change of the NM with respect to $p$ for fixed $\phi(\phi=0)(\mathbf{a})$, and the change of NM with respect to $\phi$ for fixed $p(p=0.4)(\mathbf{b})$. For both plots $T=\omega_{E}=1, \gamma=\frac{\pi}{14}$ and $\delta=\frac{\pi}{6}$ which is the Markovian region of coupling presented in Fig. 2 

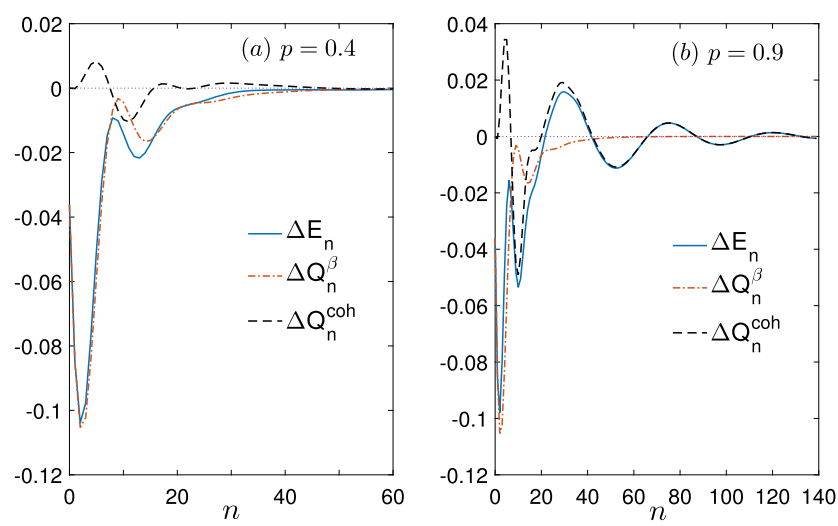

Figure $4 \Delta E_{n}, \Delta Q_{n}^{\beta}$ and $\Delta Q_{n}^{\text {coh }}$ with respect to $n$ for fixed $\phi(\phi=0), T=\omega_{E}=1, \gamma=\frac{\pi}{14}$ and $\delta=\frac{\pi}{6}$ which is the Markovian region of coupling presented in Fig. 2. $p=0.4$ of the Markovian regime (a), $p=0.9$ of the non-Markovian regime (b), and the initial state of system is excited state $|0\rangle$
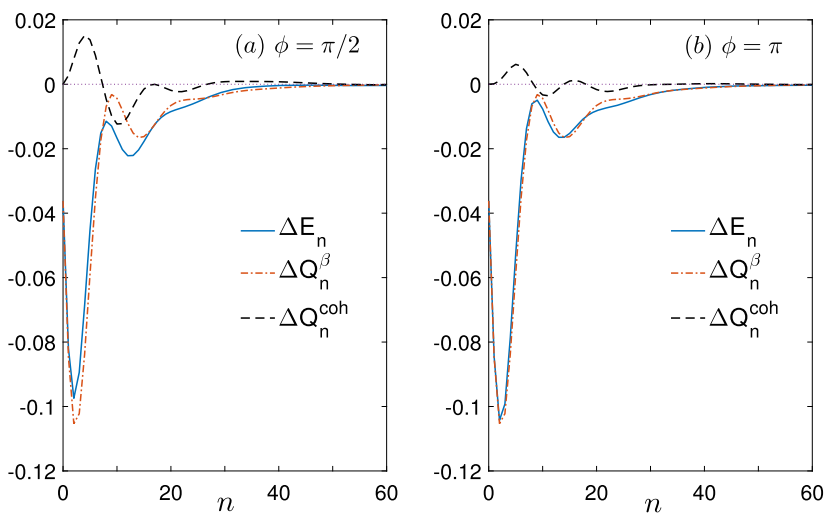

Figure $5 \Delta E_{n}, \Delta Q_{n}^{\beta}$ and $\Delta Q_{n}^{\text {coh }}$ with respect to $n$ for fixed $p(p=0.4), T=\omega_{E}=1, \gamma=\frac{\pi}{14}$ and $\delta=\frac{\pi}{6}$ which is the Markovian region of coupling presented in Fig. 2. $\phi=\pi / 2$ (a) and $\phi=\pi(\mathbf{b})$ which are the non-Markovian regime presented in Fig. $3(\mathrm{~b})$, and the initial state of system is excited state $|0\rangle$

parameter $p$. Therefore, $\Delta E_{n}$ in Eq. (17) can be divided into two parts:

$$
\Delta E_{n}=\Delta Q_{n}^{\beta}+\Delta Q_{n}^{\text {coh }}
$$

where the first term, $\Delta Q_{n}^{\beta}$, is the contribution of the first term in Eq. (16), i.e., the thermal state of environment, and the second term $\Delta Q_{n}^{\text {coh }}$ is the contribution of coherence of environment (the second term in Eq. (16)). In Fig. 4, we plot $\Delta E_{n}, \Delta Q_{n}^{\beta}$ and $\Delta Q_{n}^{\text {coh }}$ with respect to $n$ in two cases: $p=0.4$ of the Markovian regime (Fig. 4(a)) and $p=0.9$ of nonMarkovian regime (Fig. 4(b)). It shows that in the Markovian regime $\Delta Q_{n}^{\beta}$ plays a major role in $\Delta E_{n}$, which suppress energy backflow from the environment to system. In contrast to this, in the non-Markovian regime $\Delta Q_{n}^{\text {coh }}$ plays a major role of the contribution to $\Delta E_{n}$ and the energy backflow appears.

In order to study the relationship between the energy backflow of interest and the NM of the system dynamics definitely, we consider the effect of $\phi$ on NM presented in Fig. 3(b). In Fig. 5, we plot $\Delta E_{n}, \Delta Q_{n}^{\beta}$ and $\Delta Q_{n}^{\text {coh }}$ with respect to $n$ for different $\phi, \phi=\pi / 2$ (a) and 
$\phi=\pi$ (b) which are in the non-Markovian regime. We find that for the non-Markovian regime caused by $\phi, \Delta Q_{n}^{\beta}$ plays a major role in Eq. (18), and the energy flows from $S$ to its environment unidirectionally, i.e., energy backflow is suppressed. Above all, in the Markovian regime energy backflow is suppressed. However the opposite is in general not true; namely, the absence of energy backflow does not imply absence of information backflow. Note that similar results have been obtained that NM allows for the observation of energy backflow [78], and the information backflow from the reservoir to the system does not necessarily correlate with the backflow of heat [79].

\subsubsection{Effect of initial system-environment correlations on NM}

Compared to the cases discussed above without any initial correlation between system and its environment (i.e., product state), we consider a group of two initial states

$$
\begin{aligned}
\rho_{S, A_{Q}}^{1}(0) & =|\psi\rangle\langle\psi|=\left(\begin{array}{cccc}
0 & 0 & 0 & 0 \\
0 & |\xi|^{2} & \xi \sqrt{1-\xi^{2}} & 0 \\
0 & \xi \sqrt{1-\xi^{2}} & 1-|\xi|^{2} & 0 \\
0 & 0 & 0 & 0
\end{array}\right), \\
\rho_{S, A_{Q}}^{2}(0) & =|\xi|^{2}|0\rangle\langle 0|\otimes| 1\rangle\left\langle 1\left|+\left(1-|\xi|^{2}\right)\right| 1\right\rangle\langle 1|\otimes| 0\rangle\langle 0| \\
& =\left(\begin{array}{cccc}
0 & 0 & 0 & 0 \\
0 & |\xi|^{2} & 0 & 0 \\
0 & 0 & 1-|\xi|^{2} & 0 \\
0 & 0 & 0 & 0
\end{array}\right),
\end{aligned}
$$

where $|\psi\rangle=|\xi||01\rangle+\sqrt{1-|\xi|^{2}}|10\rangle$. It is worth noting that the reduced density matrixs of these two initial states are identical, and $\rho_{S, A_{Q}}^{1}(0)$ has quantum correlations (quantum entanglement and quantum discord) between $S$ and $A_{Q}$ initially, $\rho_{S, A_{Q}}^{2}(0)$ has classical correlation. We adopt Wootter's concurrence [80] as the entanglement measure. For the Xstructure density matrix $\rho_{A B}$ of bipartite system the concurrence is given by

$$
C\left(\rho_{A B}\right)=2 \max \left(0,\left|\rho_{23}\right|-\sqrt{\rho_{11} \rho_{44}},\left|\rho_{14}\right|-\sqrt{\rho_{22} \rho_{33}}\right)
$$

where $\rho_{i i}(i=1,2,3,4)$ are the matrix elements of $\rho_{A B}$. For the $\mathrm{X}$ state described by the density matrix $\rho_{A B}$, the analytic expression of quantum discord has been reported [81] and expressed by

$$
\mathrm{QD}\left(\rho_{A B}\right)=\min \left(Q_{1}, Q_{2}\right)
$$

where $Q_{j}=H\left(\rho_{11}+\rho_{33}\right)+\sum_{i=1}^{4} \lambda_{i} \log _{2} \lambda_{i}+D_{j}$ with $\lambda_{i}$ being the four eigenvalues of $\rho_{A B}, \quad D_{1}(\tau)=H(\tau), \quad D_{2}(\tau)=-\sum_{i=1}^{4} \rho_{i i} \log _{2} \rho_{i i}-H\left(\rho_{11}+\rho_{33}\right)$ with $\tau=(1+$ $\left.\sqrt{\left[1-2\left(\rho_{33}+\rho_{44}\right)\right]^{2}+4\left(\left|\rho_{14}\right|+\left|\rho_{23}\right|\right)^{2}}\right) / 2$ and $H(\tau)=-\tau \log _{2} \tau-(1-\tau) \log _{2}(1-\tau)$. And the classical correlation can be expressed as

$$
C_{\mathrm{cla}}\left(\rho_{A B}\right)=I\left(\rho_{A B}\right)-\mathrm{QD}\left(\rho_{A B}\right),
$$

where $I\left(\rho_{A B}\right)=S\left(\rho_{A}\right)+S\left(\rho_{B}\right)-S\left(\rho_{A B}\right)$ is the mutual information, $S(\rho)=-\operatorname{Tr} \rho \log _{2} \rho$ is the von Neumann entropy. And in the case of initial correlation between $S$ and $A_{Q}$, the non- 

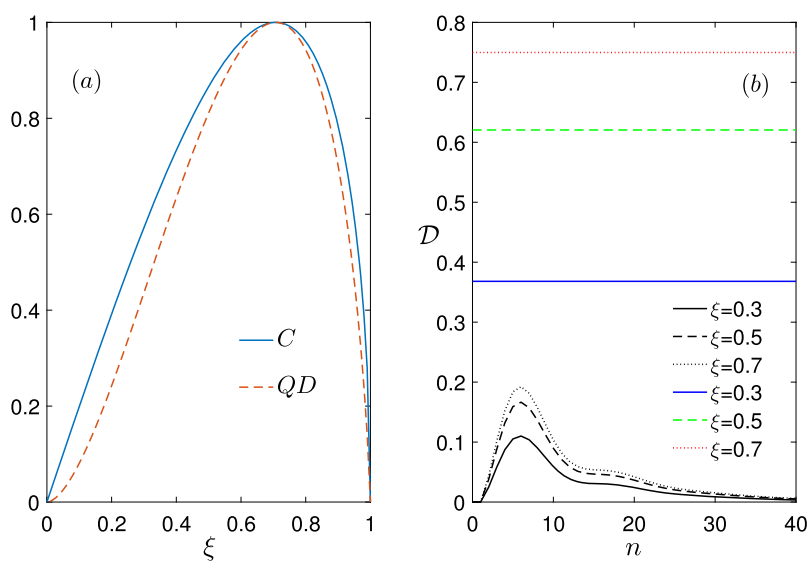

Figure 6 Concurrence (solid line) and quantum discord (dashed line) with respect to $\xi$ for initial quantum correlation Eq. (19) (a). Trace distance Eq. (23) against the number of collisions $n$ with different $\xi$ in the Markovian coupling region of Fig. $2\left(\gamma=\frac{\pi}{14}, \delta=\frac{\pi}{6}\right)$, a non-Markovian process is induced by initial quantum correlation Eq. (19), and the bound of Eq. (24) is indicated by the upper horizontal line (b)

Markovian dynamics of system can be witnessed by the non-monotonicity in evolution of trace distance between two states of system

$$
\mathcal{D}\left(\rho_{S}(n+1), \tilde{\rho}_{S}(n+1)\right) \text {. }
$$

Here $\rho_{S}(n+1)$ and $\tilde{\rho}_{S}(n+1)$ are a pair of states of system after the $(n+1)$ th step, which corresponds to the pair of initial states of the composite system ' $S-A_{Q}$ ', $\left\{\rho_{S, A_{Q}}(0), \tilde{\rho}_{S, A_{Q}}(0)\right\}$, and $\tilde{\rho}_{S, A_{Q}}(0)=\operatorname{Tr}_{A_{Q}}\left(\rho_{S, A_{Q}}(0)\right) \otimes \operatorname{Tr}_{S}\left(\rho_{S, A_{Q}}(0)\right)$. And in this section we suppose that each ancilla in $\mathcal{R}$ are initially in the thermal state $\rho_{\beta}$ with $T=\omega_{E}=1$.

From Eqs. (21) and (22), the amount of entanglement and quantum discord may change with $\xi$ and this change is showed in Fig. 6(a). They (entanglement and quantum discord) have the similar behaviors, and in the region $\xi \in[0,0.7]$ the amount of entanglement and quantum discord increase with the increase of $\xi$. In Fig. 6(b) we plot the trace distance Eq. (24) against the number of collisions $n$ for initial state (19) and thermal state of each ancilla of the reservoir for different $\xi(\xi=0.3,0.5,0.7), \gamma=\frac{\pi}{14}$ and $\delta=\frac{\pi}{6}$ which correspond to a Markovian region of coupling presented in Fig. 2. It shows that the trace distance increases from zero to a maximum and then decreases until to zero, which implies that a non-Markovian dynamics of system, and the amount of information backflow increases with the increase of initial quantum correlation (entanglement and quantum discord) between system and environment. It is noted that the trace distance here exceeds the initial value. Laine et al. have pointed out that the trace distance between two states of the open system can increase above its initial value when system and its environment are initially correlated [32]. And in our case it can be written as

$$
\begin{aligned}
& \mathcal{D}\left[\rho_{S}(n), \tilde{\rho}_{S}(n)\right] \\
& \quad \leq \mathcal{D}\left[\rho_{S, A_{Q}}^{1}(0), \operatorname{Tr}_{A_{Q}}\left(\rho_{S, A_{Q}}^{1}(0)\right) \otimes \operatorname{Tr}_{S}\left(\rho_{S, A_{Q}}^{1}(0)\right)\right],
\end{aligned}
$$

where $\rho_{S}(n)$ and $\tilde{\rho}_{S}(n)$ are the reduced state of system after the $n$th interaction corresponding to the initial state $\rho_{S, A_{Q}}^{1}(0)$ and $\operatorname{Tr}_{A_{Q}}\left(\rho_{S, A_{Q}}^{1}(0)\right) \otimes \operatorname{Tr}_{S}\left(\rho_{S, A_{Q}}^{1}(0)\right)$, respectively. This 
inequality shows how far from each other two initially indistinguishable reduced states can evolve when only one of the two initial states is correlated. And physically this can be understood as following: The maximal amount of information the open system can gain from the environment is the amount of information flowed out earlier from the system since the initial time, plus the information which is initially outside the open system. Thus the increase of the trace distance is bounded from above by the correlations in the initial state. We calculate the bound of Eq. (25) in different cases of $\xi$ which is showed in Fig. 6(b), and the inequality Eq. (25) is well satisfied. We notice that the maximum value of the trace distance at a certain $n$ in Fig. 6(b) is much smaller than the bound of Eq. (25) for a fixed $\xi$, i.e., the bound of Eq. (25) is actually loose. This means that only less of the information in the composite system ' $S+A_{Q}$ ' initially transfers to the reduced system $S$ during the evolution, and this is due to the Markovian reservoir $\mathcal{R}$. Moreover, Smirne et $a l$. have provided experimental evidence that if the environmental state is fixed, the trace distance between two states of an open quantum system can increase over its initial value only in the presence of initial correlations [33].

From the discussion above, it is always able to induce a transition from Markovian to non-Markovian dynamics for initial quantum correlation between system and its environment. For initial classical correlation state (20) and a thermal state of the reservoir, from numerical calculation we find that the trace distance Eq. (24) is always zero with the number of collisions $n$ within the Markovian region of coupling presented in Fig. 2. In order to study the effect of initial classical correlation on NM more comprehensively, we use the measure of the degree of NM in the Appendix (Eq. (32)), and we find the similar result that $\mathcal{N}$ in Eq. (32) is also zero. However it is worth noting that Eq. (32) can only be used to witness the occurrence of non-Markovian dynamics rather than to confirm a Markovian dynamics. Therefore, from now on it cannot guarantee that the dynamics of system must be Markovian for initial state (20). And thus for initial classical correlation we do only claim that the $\mathcal{N}$ in Eq. (32) is zero in the case of thermal reservoir comparing to the case of reservoir with coherence (see below). In Fig. 7, we plot the trace distance Eq. (24)
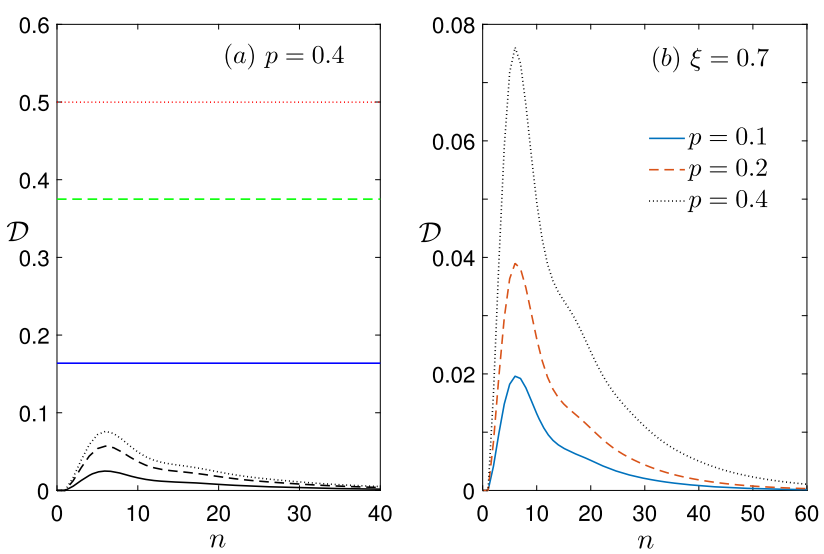

Figure 7 (a): Trace distance Eq. (24) against the number of collisions $n$ for different $\xi$, $\xi=0.3$ (black-solid line), $\xi=0.5$ (black-dashed line) and $\xi=0.7$ (black-dotted line), a non-Markovian process is induced by means of initial classical correlation Eq. (20) with coherence of reservoir, $p=0.4$ in Eq. (16) which is a Markovian regime presented in Fig. 3; and the bound of Eq. (25) is indicated by the upper blue-solid line ( $\xi=0.3)$, green-dashed line $(\xi=0.5)$ and red-dotted line $(\xi=0.7)$. (b): Trace distance Eq. (24) against the number of collisions $n$ for fixed $\xi(\xi=0.7)$ and different $p, p=\{0.1,0.2,0.4\}$. For both plots $\gamma=\frac{\pi}{14}$ and $\delta=\frac{\pi}{6}$ 
against the number of collisions $n$ for initial classical correlation state $(20) ; \gamma=\frac{\pi}{14}, \delta=\frac{\pi}{6}$, and a state with coherence (Eq. (16) with $p \neq 0$ ) of each ancilla of the reservoir, $p=0.4$ in Fig. 7(a) and $p=\{0.1,0.2,0.4\}$ in Fig. 7 (b) which corresponds to a Markovian regime presented in Fig. 3(a). Obviously the change of trace distance is similar to the case of initial quantum correlation presented in Fig. 6(b), the trace distance increases from zero to a maximum and then decreases until to zero. This also indicates a non-Markovian dynamics of system and the trace distance here exceeds the initial value. And the amount of information backflow can be increased by two ways, on the one hand with the increase of initial classical correlation between system and environment, on the other hand with the increase of coherence of reservoir. Here from numerical calculation we find that the change of amount of classical correlation with $\xi$ in Eq. (20) is the same to quantum correlation, i.e., $C_{\mathrm{cla}}\left(\rho_{S, A_{Q}}^{2}(0)\right)$ increases with the increase of $\xi$ in the region $\xi \in[0,0.7]$. Note that in Ref. [32] it has pointed out that the effects of initial classical correlation are related to the form of interaction, and they have verified that the existence of the initial classical correlation will not make the trace distance of the system exceed the initial value if two qubits are under the action of controlled-NOT gate only; and if first apply the controlled-NOT gate and then a swap operation, it can obtain a growth of the trace distance. In our case, a growth of the trace distance and a non-Markovian dynamics are emerged by means of coherence of reservoir in the case of initial classical correlation. And Eq. (25) is also satisfied now.

In summary, we study the effect of initial system-environment correlations on system dynamics, including quantum correlation and classical correlation. We realize a growth of the trace distance and a non-Markovian dynamics with the help of initial quantum correlation, however for initial classical correlation this can only be confirmed to occur when there is coherence of the reservoir simultaneously.

\subsection{Effect of NM on thermodynamic properties}

In this section we consider the system in contact with a thermal environment, i.e., the initial state of each ancilla of the reservoir is thermal state $\rho_{\beta}$, and the reduced state $\rho_{n}^{A_{Q}}$

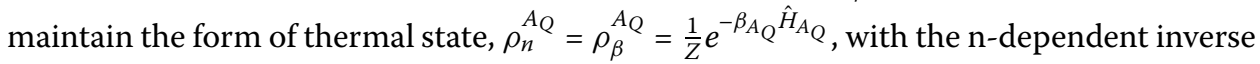
temperatures $\beta_{A_{Q}}=\frac{1}{T_{A_{Q}}}$.

Entropy change and heat flux It is known that the total von Neumann entropy of the composite system ' $S-A_{Q}$ ' under the unitary evolution $U_{S, A_{Q}}$ is invariant during each step, i.e., $S\left(\rho_{n}^{S, A_{Q}}\right)=S\left(\tilde{\rho}_{n+1}^{S, A_{Q}}\right)$, here $\tilde{\rho}_{n+1}^{S, A_{Q}}=U_{S, A_{Q}}(\gamma)\left(\rho_{n}^{S, A_{Q}}\right) U_{S, A_{Q}}^{\dagger}(\gamma)$. Based on this, the change in entropy of system during the $(n+1)$ th interaction can be expressed as [61]

$$
\begin{aligned}
\Delta \mathrm{S}_{\mathrm{n}+1} & =\mathrm{S}\left(\tilde{\rho}_{n+1}^{S}\right)-\mathrm{S}\left(\rho_{n}^{S}\right) \\
& =D\left(\tilde{\rho}_{n+1}^{S, A_{Q}} \| \tilde{\rho}_{n+1}^{S} \rho_{\beta}^{A_{Q}}\right)+\operatorname{Tr}_{A_{Q}}\left(\tilde{\rho}_{n+1}^{A_{Q}}-\rho_{\beta}^{A_{Q}}\right) \ln \rho_{\beta}^{A_{Q}}-I\left(\rho_{n}^{S, A_{Q}}\right),
\end{aligned}
$$

where $\tilde{\rho}_{n+1}^{S}=\operatorname{Tr}_{A_{Q}}\left[\tilde{\rho}_{n+1}^{S, A_{Q}}\right], \tilde{\rho}_{n+1}^{A_{Q}}=\operatorname{Tr}_{S}\left[\tilde{\rho}_{n+1}^{S, A_{Q}}\right], D\left(\rho_{1} \| \rho_{2}\right) \equiv \operatorname{Tr}\left(\rho_{1} \ln \rho_{1}\right)-\operatorname{Tr}\left(\rho_{1} \ln \rho_{2}\right)$ is the quantum relative entropy between two density matrices $\rho_{1}$ and $\rho_{2}$, and the mutual information $I\left(\rho_{n}^{S, A_{Q}}\right)=\mathrm{S}\left(\rho_{n}^{S}\right)+\mathrm{S}\left(\rho_{n}^{A_{Q}}\right)-\mathrm{S}\left(\rho_{n}^{S, A_{Q}}\right)$, measures the correlation between $S$ and $A_{Q}$, and this correlation has been established after their collision in the first step. According to the definition of $\rho_{\beta}^{A_{Q}}$ above, we can obtain $\operatorname{Tr}_{A_{Q}}\left[\left(\tilde{\rho}_{n+1}^{A_{Q}}-\rho_{\beta}^{A_{Q}}\right) \ln \rho_{\beta}^{A_{Q}}\right]=\beta_{A_{Q}} \Delta Q_{n+1}$, 

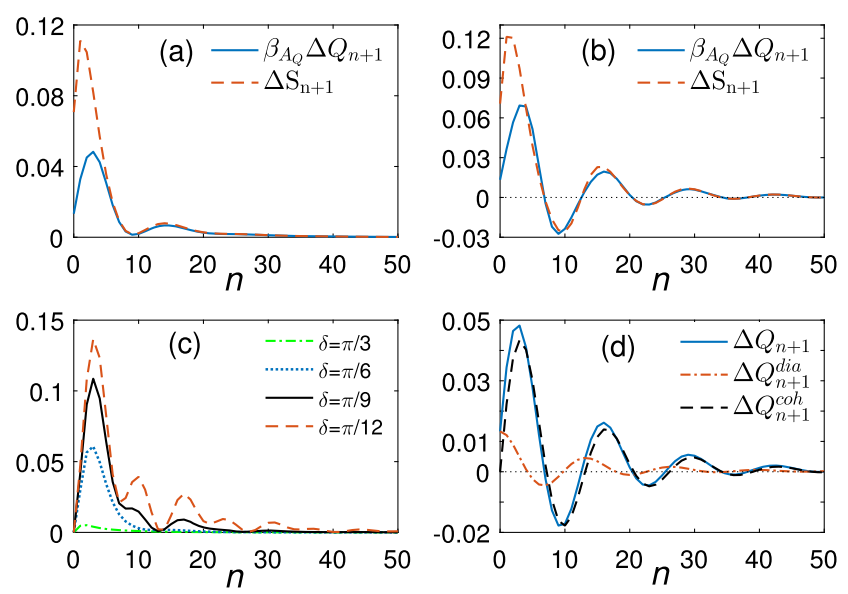

Figure 8 (a)-(b): $\Delta S_{n}$ and $\beta_{A_{Q}} \Delta Q_{n+1}$ against the number of collision $n$ with $\gamma=\frac{\pi}{14}$ and different $\delta, \delta=\frac{\pi}{6}$ (Markovian region of coupling $(\mathbf{a})), \delta=\frac{\pi}{9}$ (non-Markovian region of coupling $\left.(\mathbf{b})\right)$. (c) The correlation $/\left(\rho_{n}^{S, A} Q_{Q}\right.$ ) in Eq. (28) against the number of collision $n$ with fixed $\gamma\left(\gamma=\frac{\pi}{14}\right)$ and different $\delta$. (d) The heat $\Delta Q_{n+1}$ absorbed by system and its two contributions $\Delta Q_{n+1}^{\text {dia }}$ and $\Delta Q_{n+1}^{\text {coh }}$ against the number of collision $n$, and the parameters are the same as those of $(\mathbf{b})$. For all plots the initial state of system is ground state $|1\rangle, A_{Q}$ and each ancilla of the reservoir are initially prepared in the same thermal states $\rho_{\beta}$, i.e., Eq. (16) with $p=0, T=1$ and $\omega=1$

here

$$
\Delta Q_{n+1}=\operatorname{Tr}_{A_{Q}}\left[\left(\rho_{n}^{A_{Q}}-\tilde{\rho}_{n+1}^{A_{Q}}\right) \hat{H}_{A_{Q}}\right]
$$

representing the heat flowing from auxiliary qubit $A_{Q}$ to system $S$. Therefore, Eq. (26) can also be written as

$$
\Delta \mathrm{S}_{\mathrm{n}+1}=D\left(\tilde{\rho}_{n+1}^{S, A_{Q}} \| \tilde{\rho}_{n+1}^{S} \rho_{\beta}^{A_{Q}}\right)+\beta_{A_{Q}} \Delta Q_{n+1}-I\left(\rho_{n}^{S, A_{Q}}\right)
$$

Notice that we choose energy-preserving interactions between the bipartite systems, ' $S$ $A_{Q}$,' ' $A_{Q}-\mathcal{R}_{j}$.' Mathematically, this translates as $\left[\hat{U}_{S, A_{Q}}, \hat{H}_{S, A_{Q}}^{\mathrm{fre}}\right]=0,\left[\hat{V}_{A_{Q}, \mathcal{R}_{j}}, \hat{H}_{A_{Q}, \mathcal{R}_{j}}^{\mathrm{fre}}\right]=0$, that is, $\left[\hat{H}_{S, A_{Q}}^{\text {int }}, \hat{H}_{S, A_{Q}}^{\text {fre }}\right]=0,\left[\hat{H}_{A_{Q}, \mathcal{R}_{j}}^{\text {int }}, \hat{H}_{A_{Q}, \mathcal{R}_{j}}^{\text {fre }}\right]=0$, here $\hat{H}_{S, A_{Q}}^{\text {fre }}=\hat{H}_{S}+\hat{H}_{A_{Q}}, \hat{H}_{A_{Q}, \mathcal{R}_{j}}^{\text {fre }}=\hat{H}_{A_{Q}}+\hat{H}_{\mathcal{R}_{j}}$. So that the heat given by the system is completely transferred to the environment, and vice versa. In other words, no heat is given or taken in the form of thermodynamic work while performing the unitary operations. Thus the canonical definition of heat flow $\Delta Q_{n+1}$ in Eq. (27) is valid and compatible with thermodynamics, and the term $\beta_{A_{Q}} \Delta Q_{n+1}$ in Eq. (28) is associated with the system entropy change due to heat exchanges, i.e., entropy flux.

In order to study the system entropy change especially that results from heat exchanges with the environment, in Fig. 8(a)-(b) we plot $\Delta S_{n+1}$ and $\beta_{A_{Q}} \Delta Q_{n+1}$ against the number of collisions $n$ of a Markovian region of coupling $\left(\gamma=\frac{\pi}{14}, \delta=\frac{\pi}{6}\right)$ in Fig. 8(a) and a nonMarkovian region of coupling $\left(\gamma=\frac{\pi}{14}, \delta=\frac{\pi}{9}\right)$ in Fig. 8(b). The initial state of system is ground state $|1\rangle$, and the initial states of auxiliary qubit and reservoir qubits are in the same thermal state $\rho_{\beta}\left(p=0\right.$ in Eq. (16)) with $T=1$. It shows that the changes of $\Delta S_{n+1}$ and $\beta_{A_{Q}} \Delta Q_{n+1}$ are almost consistent with the increase of $n$, increasing first and then oscillating decay. However $\Delta S_{n+1}$ and $\beta_{A_{Q}} \Delta Q_{n+1}$ are always larger than zero for Markovian environment (Fig. 8(a)), and which can be less than zero during some time intervals for non-Markovian environment (Fig. 8(b)). Physically this can be understood as following. 
We define $\rho_{i j}(i, j=1,2,3,4)$ are the matrix elements of state $\rho_{n}^{S, A_{Q}}$ of ' $S-A_{Q}$ ' before their $(n+1)$ th collisions. Due to the correlations between $S$ and $A_{Q}, \Delta Q_{n+1}$ in Eq. (27) can be divided into two different contributions:

$$
\Delta Q_{n+1}=\Delta Q_{n+1}^{\mathrm{dia}}+\Delta Q_{n+1}^{\mathrm{coh}}
$$

where

$$
\begin{aligned}
& \Delta Q_{n+1}^{\text {dia }}=\omega \sin ^{2}(\gamma)\left(\rho_{33}-\rho_{22}\right), \\
& \Delta Q_{n+1}^{\text {coh }}=\omega \operatorname{Im}\left(\rho_{23}\right) \sin (2 \gamma),
\end{aligned}
$$

are the heats determined, respectively, by the diagonal and coherent (off-diagonal) elements of state $\rho_{n}^{S, A_{Q}}$, and $\omega=\omega_{S}=\omega_{E}$ is the resonance frequency of $S, A_{Q}$ and $\mathcal{R}_{j}$. The nonzero coherent term $\rho_{23}$ of $\rho_{n}^{S, A Q}$ is a direct witness of correlation between $S$ and $\rho_{n}^{A_{Q}}$, which in turn gives the correlation-dependent heat $\Delta Q_{n+1}^{\text {coh }}$. For fixed parameter $\gamma$, the relatively large values of $\delta$ lead to Markovian dynamics, and the established systemenvironment correlations are weak, so the contribution $\Delta Q_{n+1}^{\text {dia }}$ plays a major role in determining the behavior of total heat $\Delta Q_{n+1}$. This can be verified by Fig. 8(c): the correlations $I\left(\rho_{n}^{S, A_{Q}}\right)$ established within the dynamical process decrease with the increase of $\delta$ for fixed $\gamma$. Differently, when $\delta$ is sufficiently small (non-Markovian dynamics) the behavior of $\Delta Q_{n+1}$, especially its transition from positive to negative values, is mainly determined by the contribution $\Delta Q_{n+1}^{\text {coh }}$, as showed in Fig. 8(d).

Irreversible entropy production The entropy production can be defined as the difference between the change in entropy of the reduced system state and the mean exchanged heat with a reservoir at fixed temperature, $T$, divided by $T[66,69,71]$. From Eq. (28), the irreversible contribution to the entropy production during the $(n+1)$ th interaction can be written as

$$
\begin{aligned}
\Sigma_{n+1} & =\Delta \mathrm{S}_{\mathrm{n}+1}-\beta_{A_{Q}} \Delta Q_{n+1} \\
& =D\left(\tilde{\rho}_{n+1}^{S, A_{Q}} \| \tilde{\rho}_{n+1}^{S} \rho_{\beta}^{A_{Q}}\right)-I\left(\rho_{n}^{S, A_{Q}}\right),
\end{aligned}
$$

it provides the contribution in entropy change of system which cannot be traced back to a reversible heat flow. In Fig. 9, we plot entropy production of system $\Sigma, D\left(\tilde{\rho}_{n+1}^{S, A_{Q}} \| \tilde{\rho}_{n+1}^{S} \rho_{\beta}^{A_{Q}}\right)$ (the first term in Eq. (31)) and the established system-environment correlation $I\left(\rho_{n}^{S, A_{Q}}\right)$ (the second term in Eq. (31)), with respect to $n$ for different dynamics of system. As expected, we find the entropy production of system can become transiently negative for the non-Markovian dynamics compared with the corresponding Markovian case. In other words, in some specific time intervals the entropy production can decrease, provided that the quantum dynamics fails to be positive divisible, i.e. it is essentially non-Markovian. And the multiple-interaction entropy production, $\Sigma$ is zero regardless of whether the underlying dynamics is Markovian or non-Markovian, which is due to thermalization of system with the environment, i.e., $S$ is in a thermal equilibrium state $\rho_{\beta}$ in the long-time limit.

In Fig. 10, we study the entropy production of system $\Sigma$ against the number of collisions $n$ for initial quantum correlation between the system and its environment Eq. (19). We 

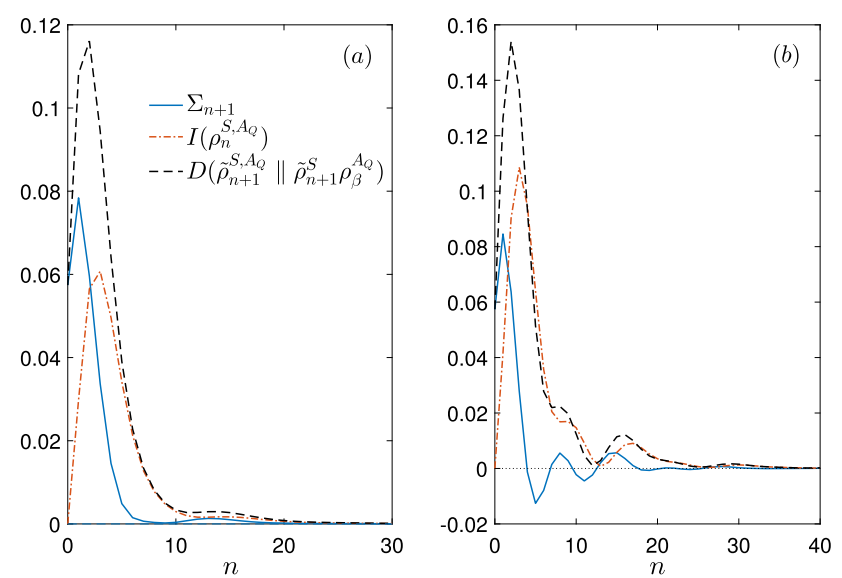

Figure 9 Behaviors of entropy production of system $\Sigma$, the established system-environment correlation $I\left(\rho_{n}^{S, A} Q\right)$, and $D\left(\tilde{\rho}_{n+1}^{S, A} \| \tilde{\rho}_{n+1}^{S} \rho_{\beta}^{A_{Q}}\right)$, for non-Markovian dynamics $\left(\gamma=\frac{\pi}{14}\right.$ and $\left.\delta=\frac{\pi}{9}\right)(\mathbf{b})$ compared with the corresponding Markovian case $\left(\gamma=\frac{\pi}{14}\right.$ and $\left.\delta=\frac{\pi}{6}\right)$ (a). For both plots the system is in ground state $|1\rangle$ initially, and initial thermal state of each element of the environment, $T=1$ and $\omega=1$
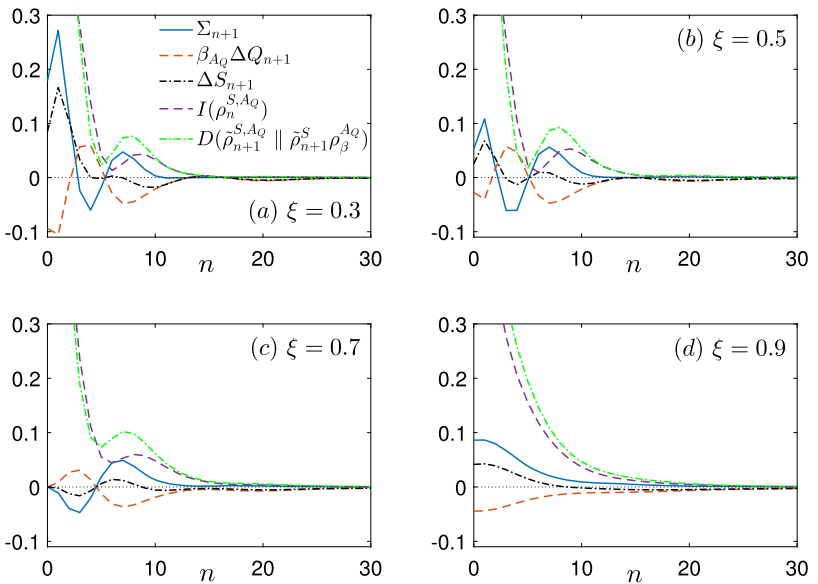

Figure 10 Behaviors of entropy production of system $\Sigma, \Delta S_{n}$ and $\beta_{A_{Q}} \Delta Q_{n+1}, I\left(\rho_{n}^{S, A}{ }^{S}\right)$ and $D\left(\tilde{\rho}_{n+1}^{S, A} Q \| \tilde{\rho}_{n+1}^{S} \rho_{\beta}^{A Q}\right)$, for initial quantum correlation between the system and its environment Eq. (19) regarding different $\xi$, and initial thermal state of each ancilla of the reservoir. And for all plots $\gamma=\frac{\pi}{14}, \delta=\frac{\pi}{6}$, $T=1$ and $\omega=1$

find that it can undergoes a negative $\Sigma$ during the dynamics in some cases, for example, $\xi=\{0.3,0.5,0.7\}$ in Eq. (19). However $\Sigma$ can always positive during the whole dynamics for $\xi=0.9$. In other words, we see the possibility of positive $\Sigma$ induced by the kind of NM of the initial quantum correlation. And this is different from the kind of NM induced by the coupling strength of system-auxiliary system and auxiliary system-reservoir mentioned above, that there is a corresponding relationship between non-Markovian dynamics and a negative $\Sigma$. Consequently, the NM originated from the coupling strength induces a negative $\Sigma$ definitely, whereas from the initial quantum correlation may be positive or negative. And a similar result has been obtained that the non-Markovian effect regarding the initial correlation may yield positive entropy production rate [71]. 


\section{Conclusion}

In this paper, we have investigated the non-Markovian character of the system and its effect on thermodynamic properties by means of a collision model, that a system is coupled to a structured environment consisting of a auxiliary system and a reservoir. We have studied how the system-auxiliary system and auxiliary system-reservoir coupling strength, coherence of environment and initial system-environment correlation affect the nonMarkovian character of the system. Especially we have studied the non-Markovian dynamics induced by coherence of environment from the perspective of energy, and the relationship between information backflow and energy flux. And we have shown the growth of trace distance regarding initial classical correlation between the system and its environment by means of the coherence of reservoir, and this is different from the result showed in Ref. [32] that the effect of initial classical correlation on the growth of trace distance is related to the form of interaction.

Then we have studied the effects of NM on the entropy change of the system. We have shown that the essence of entropy flux (the system entropy change induced by heat exchange with the environment) between positive and negative values under non-Markovian evolution is due to the contribution of heat flux induced by coherence. And we have observed a one-to-one correspondence between a transient negative values of the entropy production and non-Markovian dynamics induced by the coupling strength. On the contrast, we have shown the possibility of positive entropy production during the whole nonMarkovian dynamics induced by initial system-environment correlation.

Note that in this paper we have used the collision model to investigate the influences of non-Markovian dynamics, and the relation of NM and thermodynamics. The reason to consider this simple model is that exact solutions can be obtained for a general class of initial system-environment correlations and the initial states of reservoirs with coherence. We expect that some features of the NM and thermodynamics in this simple model might be similar to those in more involved but less tractable structured-environment models, so we can gain some insight into the general feature of the effects of initial systemenvironment correlations and reservoirs with coherence on NM, and hence the relation between NM and thermodynamic properties.

\section{Appendix: NM witness with initial classical correlation}

We introduce the degree of NM which makes use of the non-monotonicity of the trace distance between two states of system to witness the effect of initial classical correlation on the non-Markovian dynamics of the system [54],

$$
\mathcal{N}=\max _{\theta \in[0, \pi], \varphi \in[0,2 \pi]} \sum_{n \in \sigma^{+}} \Delta \mathcal{D}(n+1)
$$

where $\Delta \mathcal{D}(n+1)=\mathcal{D}\left[\rho_{s}(n+1), \tilde{\rho}_{s}(n+1)\right]-\mathcal{D}\left[\rho_{s}(n), \tilde{\rho}_{s}(n)\right]$, and $\rho_{s}(n+1)$ is the same as that in Eq. (19), $\tilde{\rho}_{s}(n+1)$ is the reduced state of system after the $(n+1)$ th interaction corresponding to the initial state $\rho_{S, A_{Q}}(0)=\tilde{\rho}_{s}(0) \otimes \operatorname{Tr}_{S}\left(\rho_{S, A_{Q}}^{2}(0)\right)$ with the initial system state $\tilde{\rho}_{s}(0)=\cos \frac{\theta}{2}|0\rangle+e^{i \varphi} \sin \frac{\theta}{2}|1\rangle, \theta \in[0, \pi], \varphi \in[0,2 \pi]$. The maximization is performed by taking all possible system states $\tilde{\rho}_{s}(0)$ over the Bloch sphere. And here the definition of $\sigma^{+}$is the same as that in Eq. (13), in which $\Delta \mathcal{D}(n)>0$. 


\section{Acknowledgements}

The authors are grateful to Prof. Jian Zou, Prof. Zhongxiao Man and Dr. Chaoquan Wang for fruitful discussions.

\section{Funding}

The National Natural Science Foundation of China (Grant No. 11947047).

\section{Abbreviations}

NM, Non-Markovianity.

\section{Availability of data and materials}

The data sets used or analysed during the current study are available from the corresponding author on reasonable request.

\section{Competing interests}

The authors declare that they have no competing interests.

\section{Authors' contributions}

YWL performed calculations, analysed the results and wrote the manuscript. LL originated the idea, directed the project, analysed the results and helped in writing. All authors discussed the results and commented on the manuscript. All authors read and approved the final manuscript.

\section{Publisher's Note}

Springer Nature remains neutral with regard to jurisdictional claims in published maps and institutional affiliations.

Received: 12 October 2020 Accepted: 6 April 2021 Published online: 15 April 2021

\section{References}

1. Breuer HP, Petruccione F. The theory of open quantum systems. Oxford: Oxford University Press; 2002.

2. Mogilevtsev D, Nisovtsev AP, Kilin S, Cavalcanti SB, Brandi HS, Oliveira LE. Driving-dependent damping of Rabi oscillations in two-level semiconductor systems. Phys Rev Lett. 2008;100:017401.

3. Galland C, Hogele A, Tureci HE, Imamoglu A. Non-Markovian decoherence of localized nanotube excitons by acoustic phonons. Phys Rev Lett. 2008;101:067402.

4. Madsen KH, Ates S, Lund-Hansen T, Loffler A, Reitzenstein S, Forchel A, Lodahl P. Observation of non-Markovian dynamics of a single quantum dot in a micropillar cavity. Phys Rev Lett. 2011;106:233601.

5. Tang JS, Li CF, Li YL, Zou XB, Guo GC, Breuer HP, Laine EM, Piilo J. Measuring non-Markovianity of processes with controllable system-environment interaction. Europhys Lett. 2012;97:10002.

6. Hu BL, Paz JP, Zhang Y. Quantum Brownian motion in a general environment: exact master equation with nonlocal dissipation and colored noise. Phys Rev D. 1992:45:2843.

7. Strunz WT, Yu T. Convolutionless non-Markovian master equations and quantum trajectories: Brownian motion. Phys Rev A. 2004;69:052115.

8. Vacchini B, Breuer HP. Exact master equations for the non-Markovian decay of a qubit. Phys Rev A. 2010;81:042103.

9. Zhang WM, Lo PY, Xiong HN, Tu MWY, Nori F. General non-Markovian dynamics of open quantum systems. Phys Rev Lett. 2012:109:170402.

10. Wolf MM, Eisert J, Cubitt TS, Cirac Jl. Assessing non-Markovian quantum dynamics. Phys Rev Lett. 2008;101:150402.

11. Zhang GF, Ji AL, Fan H, Liu WM. Quantum correlation dynamics of two qubits in noisy environments: the factorization law and beyond. Ann Phys. 2012;327:2074.

12. Jing J, Segal D, Li B, Wu LA. Transient unidirectional energy flow and diode-like phenomenon induced by non-Markovian environments. Sci Rep. 2015;5:15332.

13. Criger B, Ciani A, DiVincenzo DP. Multi-qubit joint measurements in circuit QED: stochastic master equation analysis. EPJ Quantum Technol. 2016;3:6.

14. Breuer HP, Laine EM, Piilo J. Measure for the degree of non-Markovian behavior of quantum processes in open systems. Phys Rev Lett. 2009:103:210401.

15. Rivas A, Huelga SF, Plenio MB. Entanglement and non-Markovianity of quantum evolutions. Phys Rev Lett. 2010;105:050403.

16. Luo S, Fu S, Song H. Quantifying non-Markovianity via correlations. Phys Rev A. 2012;86:044101.

17. Laine EM, Piilo J, Breuer HP. Measure for the non-Markovianity of quantum processes. Phys Rev A. 2010;81:062115.

18. Xu ZY, Yang WL, Feng M. Proposed method for direct measurement of the non-Markovian character of the qubits coupled to bosonic reservoirs. Phys Rev A. 2010;81:044105.

19. He Z, Zou J, Li L, Shao B. Effective method of calculating the non-Markovianity $N$ for single-channel open systems. Phys Rev A. 2011;83:012108.

20. Zeng HS, Tang N, Zheng YP, Xu TT. Non-Markovian dynamics for an open two-level system without rotating wave approximation: indivisibility versus backflow of information. Eur Phys J D. 2012;66:255.

21. Haikka P, Johnson TH, Maniscalco S. Non-Markovianity of local dephasing channels and time-invariant discord. Phys Rev A. 2013;87:010103.

22. Addis C, Haikka P, McEndoo S, Macchiavello C, Maniscalco S. Two-qubit non-Markovianity induced by a common environment. Phys Rev A. 2013:87:052109.

23. Fanchini FF, Karpat G, Castelano LK, Rossatto DZ. Probing the degree of non-Markovianity for independent and common environments. Phys Rev A. 2013;88:012105.

24. Reich DM, Katz N, Koch CP. Exploiting non-Markovianity for quantum control. Sci Rep. 2015;5:12430.

25. Liu BH, Li L, Huang YF, Li CF, Guo GC, Laine EM, Breuer HP, Piilo J. Experimental control of the transition from Markovian to non-Markovian dynamics of open quantum systems. Nat Phys. 2011;7:931. 
26. Brito F, Werlang T. A knob for Markovianity. New J Phys. 2015;17:072001.

27. Ma TT, Chen YS, Chen T, Hedemann SR, Yu T. Crossover between non-Markovian and Markovian dynamics induced by a hierarchical environment. Phys Rev A. 2014;90:042108.

28. Levi EK, Irish EK, Lovett BW. Coherent exciton dynamics in a dissipative environment maintained by an off-resonant vibrational mode. Phys Rev A. 2016;93:042109.

29. Raja SH, Borrelli M, Schmidt R, Pekola JP, Maniscalco S. Thermodynamic fingerprints of non-Markovianity in a system of coupled superconducting qubits. Phys Rev A. 2018;97:032133.

30. Xu K, Zhang YJ, Xia YJ, Wang ZD, Fan H. Hierarchical-environment-assisted non-Markovian speedup dynamics control. Phys Rev A. 2018;98:022114.

31. Dajka J, Łuczka J. Distance growth of quantum states due to initial system-environment correlations. Phys Rev A. 2010;82:012341.

32. Laine EM, Piilo J, Breuer HP. Witness for initial system-environment correlations in open-system dynamics. Europhys Lett. 2010:92:60010.

33. Smirne A, Brivio D, Cialdi S, Vacchini B, Paris MGA. Experimental investigation of initial system-environment correlations via trace-distance evolution. Phys Rev A. 2011:84:032112.

34. Scarani V, Ziman M, Štelmachovič P, Gisin N, Bužek V. Thermalizing quantum machines: dissipation and entanglement. Phys Rev Lett. 2002;88:097905.

35. Giovannetti V, Palma GM. Master equations for correlated quantum channels. Phys Rev Lett. 2012;108:040401.

36. Giovannetti V, Palma GM. Master equation for cascade quantum channels: a collisional approach. J Phys B. 2012:45:154003

37. Caruso F, Giovannetti V, Lupo C, Mancini S. Quantum channels and memory effects. Rev Mod Phys. 2014;86:1203.

38. Strasberg P, Schaller G, Brandes T, Esposito M. Quantum and information thermodynamics: a unifying framework based on repeated interactions. Phys Rev X. 2017;7:021003.

39. Seah S, Nimmrichter S, Grimmer D, Santos JP, Scarani V, Landi GT. Collisional quantum thermometry. Phys Rev Lett. 2019;123:180602.

40. Rodrigues FLS, De Chiara G, Paternostro M, Landi GT. Thermodynamics of weakly coherent collisional models. Phys Rev Lett. 2019;123:140601.

41. Rau J. Relaxation phenomena in spin and harmonic oscillator systems. Phys Rev. 1963;129:1880.

42. Alicki R, Lendi K. Quantum dynamical semigroups and applications. Lecture notes in physics. Berlin: Springer; 1987.

43. Rybár T, Filippov SN, Ziman M, Bužek V. Simulation of indivisible qubit channels in collision models. J Phys B. 2012:45:154006

44. Ciccarello F, Palma GM, Giovannetti V. Collision-model-based approach to non-Markovian quantum dynamics. Phys Rev A. 2013:87:040103.

45. Ciccarello F, Giovannetti V. A quantum non-Markovian collision model: incoherent swap case. Phys Scr T. 2013;153:014010.

46. Bernardes NK, Carvalho ARR, Monken CH, Santos MF. Environmental correlations and Markovian to non-Markovian transitions in collisional models. Phys Rev A. 2014;90:032111.

47. McCloskey R, Paternostro M. Non-Markovianity and system-environment correlations in a microscopic collision model. Phys Rev A. 2014;89:052120.

48. Kretschmer S, Luoma K, Strunz WT. Collision model for non-Markovian quantum dynamics. Phys Rev A. 2016;94:012106.

49. Çakmak B, Pezzutto M, Paternostro M, Müstecaplıoğlu ÖE. Non-Markovianity, coherence, and system-environment correlations in a long-range collision model. Phys Rev A. 2017;96:022109.

50. Bernardes NK, Carvalho ARR, Monken CH, Santos MF. Coarse graining a non-Markovian collisional model. Phys Rev A. 2017;95:032117

51. de Vega I, Alonso D. Dynamics of non-Markovian open quantum systems. Rev Mod Phys. 2017;89:15001.

52. Campbell S, Ciccarello F, Palma GM, Vacchini B. System-environment correlations and Markovian embedding of quantum non-Markovian dynamics. Phys Rev A. 2018;98:012142.

53. Lorenzo S, Ciccarello F, Palma GM. Composite quantum collision models. Phys Rev A. 2017:96:032107.

54. Wang CQ, Zou J, Shao B. Analysis of various factors affecting the non-Markovian dynamics associated with a hierarchical environment based on collision model. Quantum Inf Process. 2017:16:156.

55. Li L, Zou J, Li H, Xu BM, Wang YM, Shao B. Effect of coherence of nonthermal reservoirs on heat transport in a microscopic collision model. Phys Rev E. 2018;97:022111.

56. Jin J, Yu CS. Non-Markovianity in the collision model with environmental block. New J Phys. 2018;20:053026.

57. Li Y, Li XL, Jin JS. Information scrambling in a collision model. Phys Rev A. 2020;101:042324.

58. Lorenzo S, Farace A, Ciccarello F, Palma GM, Giovannetti V. Heat flux and quantum correlations in dissipative cascaded systems. Phys Rev A. 2015;91:022121.

59. Lorenzo S, McCloskey R, Ciccarello F, Paternostro M, Palma GM. Landauer's principle in multipartite open quantum system dynamics. Phys Rev Lett. 2015;115:120403.

60. Pezzutto M, Paternostro M, Omar Y. Implications of non-Markovian quantum dynamics for the Landauer bound. New J Phys. 2016;18:123018.

61. Man ZX, Xia YJ, Lo Franco R. Validity of the Landauer principle and quantum memory effects via collisional models. Phys Rev A. 2019;99:042106.

62. Abiuso P, Giovannetti V. Non-Markov enhancement of maximum power for quantum thermal machines. Phys Rev A. 2019:99:052106

63. Pezzutto M, Paternostro M, Omar Y. An out-of-equilibrium non-Markovian quantum heat engine. Quantum Sci Technol. 2019:4:025002.

64. Katz G, Kosloff R. Quantum thermodynamics in strong coupling: heat transport and refrigeration. Entropy. 2016;18:186.

65. Man ZX, Xia YJ, Lo Franco R. Temperature effects on quantum non-Markovianity via collision models. Phys Rev A. 2018;97:062104

66. Funo K, Ueda M, Sagawa T. Quantum fluctuation theorems. arXiv:1803.04778. 
67. Landi GT, Paternostro M. Irreversible entropy production, from quantum to classical. arXiv:2009.07668.

68. Marcantoni S, Alipour S, Benatti F, Floreanini R, Rezakhani AT. Entropy production and non-Markovian dynamical maps. Sci Rep. 2017;7:12447.

69. Popovic M, Vacchini B, Campbell S. Entropy production and correlations in a controlled non-Markovian setting. Phys Rev A. 2018;98:012130.

70. Strasberg P, Esposito M. Non-Markovianity and negative entropy production rates. Phys Rev E. 2019:99:012120.

71. Xu YY, Liu J, Feng M. Positive entropy production rate induced by non-Markovianity. Phys Rev E. 2018;98:032102.

72. Cusumano S, Cavina V, Keck M, De Pasquale A, Giovannetti V. Entropy production and asymptotic factorization via thermalization: a collisional model approach. Phys Rev A. 2018;98:032119.

73. Gherardini S, Marcantoni S, Caruso F. Irreversibility mitigation in unital non-Markovian quantum evolutions. Phys Rev Res. 2020:2:033250

74. Nielsen MA, Chuang IL. Quantum computation and quantum information. Cambridge: Cambridge University Press; 2000.

75. Ruskai MB. Beyond strong subadditivity? Improved bounds on the contraction of generalized relative entropy. Rev Math Phys. 1994;6:1147.

76. Alicki R, Lendi K. Quantum dynamical semigroups and applications. Berlin: Springer; 2007.

77. Vacchini B. A classical appraisal of quantum definitions of non-Markovian dynamics. J Phys B. 2012;45:154007.

78. Guarnieri G, Uchiyama C, Vacchini B. Energy backflow and non-Markovian dynamics. Phys Rev A. 2016;93:012118.

79. Schmidt R, Maniscalco S, Ala-Nissila T. Heat flux and information backflow in cold environments. Phys Rev A. 2016:94:010101.

80. Wootters WK. Entanglement of formation of an arbitrary state of two qubits. Phys Rev Lett. 1998;80:2245.

81. Wang CZ, Li CX, Nie LY, Li JF. Classical correlation and quantum discord mediated by cavity in two coupled qubits. J Phys B. 2011;44:015503.

\section{Submit your manuscript to a SpringerOpen ${ }^{\circ}$ journal and benefit from:}

- Convenient online submission

- Rigorous peer review

- Open access: articles freely available online

- High visibility within the field

- Retaining the copyright to your article

Submit your next manuscript at $>$ springeropen.com 